\title{
Study on the Calibration of LHD Neutron Monitoring System*)
}

\author{
Naoto NISHIO, Atsushi YAMAZAKI, Kenichi WATANABE, Akira URITANI, \\ Mitsutaka ISOBE $^{1)}$ and Hirokuni YAMANISHI ${ }^{1)}$ \\ Division of Quantum Science and Energy Engineering, Nagoya University, Nagoya 464-8603, Japan \\ ${ }^{1)}$ National Institute for Fusion Science, Toki 509-5292, Japan
}

(Received 5 January 2011 / Accepted 2 June 2011)

\begin{abstract}
Neutron monitoring is quite important because neutron yield generated by fusion reactions corresponds to the fusion output. In design of the neutron monitor, Monte Carlo simulations play an important role to make corrections on various parameters, such as neutron energy spectrum and spatial distribution when determining the calibration constant. We consider the calibration procedures using a $\mathrm{Cf}$ point source toroidally rotating in the vacuum vessel, and evaluate uncertainties of the calibration constant for the neutron detector placed on the center axis.

(C) 2011 The Japan Society of Plasma Science and Nuclear Fusion Research
\end{abstract}

Keywords: neutron monitor, MCNP, calibration, LHD, fusion reactor

DOI: $10.1585 /$ pfr.6.2405115

\section{Introduction}

Deuterium plasma experiments are in plan on Large Helical Device (LHD) at National Institute for Fusion Science (NIFS). In calibration experiments, neutrons are generated as a result of DD fusion reactions. Since fusion neutrons are a direct evidence of fusion reactions, total fusion neutron yield can be a measure of the fusion reactor output. Neutron monitoring, therefore, is quite important. The calibration experiments should be performed to connect the neutron monitor count with the total neutron yield which is corresponding to the fusion reactor output. In calibration experiments for a neutron monitoring system, Monte Carlo simulations play an important role to make corrections on various effects which are influence of neutron scattering, a difference between spectra of fusion neutrons and a $\mathrm{Cf}$ neutron source and neutron spatial distribution. Helical type fusion devices have quite complicated geometry compared to tokamak type devices $[1,2]$. Therefore, it takes a lot of work and time to construct the geometry file for a Monte Carlo simulation code [3]. So far, we made a program that can automatically generate an input file of a simplified helical coil geometry for the MCNP Monte Carlo code. By using this program, the neutron spatial distributions and neutron spectra around the device were successfully calculated [4]. In this paper, we discuss the procedures of the calibration experiments and preliminarily evaluate the

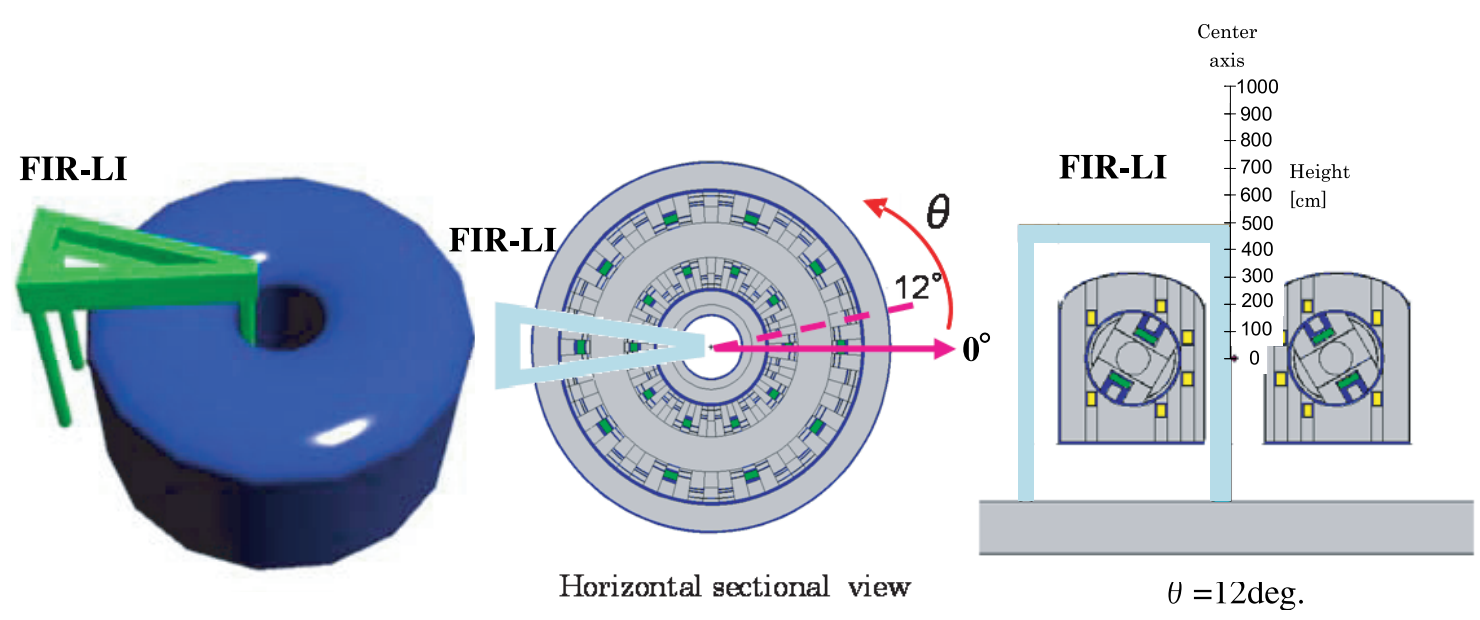

Fig. 1 Schematic view of geometry used in MCNP calculations. The origin of the vertical axis is set at the equatorial plane of the torus. The triangle structure is the support structure for the FIR laser interferometer (FIR-LI).

author'se-mail: nishio.naoto@b.mbox.nagoya-u.ac.jp

*) This article is based on the presentation at the 20th International Toki

Conference (ITC20). 
uncertainty of the calibration constant when the neutron monitor is placed on center axis.

\section{Simulation of Calibration Experi- ments}

\subsection{Geometry}

We showed the schematic view of the geometry in Fig. 1. The geometry automatically generated by our program was divided with equal angle intervals in the toroidal direction. The helical coils were imitated by gradually rotating coil structures in the poloidal direction. In the geometry, the vacuum vessel, the poloidal coils, the cryostat, the support structures and the concrete floor were also placed in addition to the helical coils and the helical coil cans. The large support structure for the FIR laser interferometer was also placed near the devices.

\subsection{Detector structure}

As a neutron detector, we adopted the ${ }^{235} \mathrm{U}$ fission chamber $\left({ }^{235} \mathrm{U}: 1.5 \mathrm{~g}\right)$ that was used in JT-60U experiments. The fission chamber was surrounded by a polyethylene moderator with $70 \mathrm{~mm}$ thickness and a cadmium thermal neutron shield with $1 \mathrm{~mm}$ thickness. The moderator enhances the detection efficiency for fast neutrons. The moderator thickness was adjusted to obtain the flat-response for neutron energy.

\subsection{Simulation results}

We evaluate the absolute detection efficiency of the neutron detector placed on center axis. In calibration experiments, ${ }^{252} \mathrm{Cf}$ sources, which has broad fission spectrum with the average energy of $2.11 \mathrm{MeV}$, are generally used.

In calibration experiments of the most fusion experimental reactors, the standard neutron point source was toroidally rotated in the devices and then the detection efficiency of the neutron detector was derived. Figure 2 shows the absolute detection efficiency, which is defined as the probability of detection for a neutron emitted from

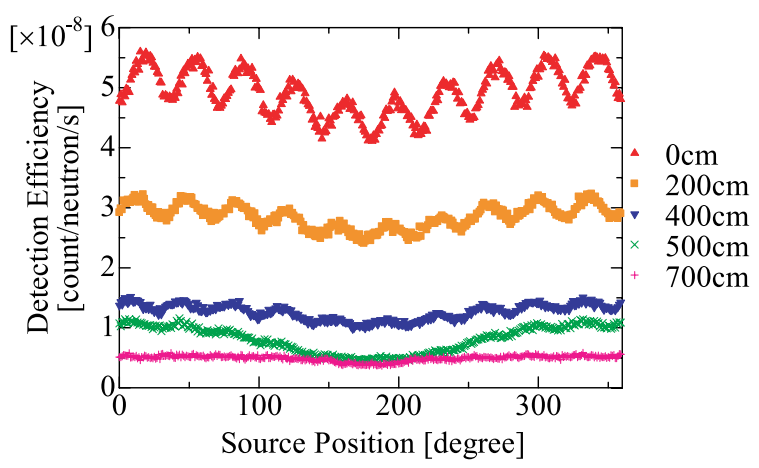

Fig. 2 Absolute detection efficiency of the neutron detector placed at various heights from an equatorial plane on the center axis of the device for a ${ }^{252} \mathrm{Cf}$ point source toroidally rotating on the torus axis of the vacuum vessel. the point source, calculated by MCNP simulation when rotating the point source with the $\mathrm{Cf}$ fission spectra in the device. To determine the height of the detector position, we calculated the detection efficiencies at various heights. The detection efficiency has 36 degree cyclic structures caused by helical coils structures and the effect of the large support structure for the FIR laser interferometer. The effect of the cyclic structure is more remarkable near the equatorial plane than higher positions.

\section{Considerations of Calibration Pro- cedures}

For calibration experiments, there are two source rotating procedures. One is the continuous source rotating method during a measurement. The other is step source rotating method where the source position is fixed during a measurement and then the point source is rotated by a certain step angle. In the latter procedure, the detector response to each source position can be obtained. We, however, should consider the source rotating procedure, such as step angle and measurement time, from the viewpoint of the total calibration time and the statistical uncertainty. We calculate the detection efficiency of the neutron detector placed on the center axis averaged over complete rotation. The relative average detection efficiency to the average one calculated by fine step angle ( $1 \mathrm{deg}$. step) are calculated for various rotation step angles. We assume the fine step angle result to be true. The results calculated by varying the rotation start position angle are plotted in Fig. 3. There is no dependence on the start position angle except for 36 degree step source rotation.

Figure 4 shows the standard deviation of the relative average detection efficiency for various rotation step angles at 500,600 and $700 \mathrm{~cm}$ on center axis. As the neutron detector position, heights less than $400 \mathrm{~cm}$ is inadequate because of the intense magnetic field and the remarkable effect of the cyclic structure of the helical coil. The standard deviation seems to increase with increasing the rotation step angle. Especially 36 and 72 degree steps have quite large deviation because the twist cycle of the helical coil of the LHD is 36 degrees. Relative standard deviations are less than 1 percent for 6 and 15 degree step and less than 5 percent for 60 degree step. Source locating precision, therefore, can be rough because the cyclic structures can be canceled except for rotation step angles synchronizing with the helical twist cycle.

\section{Uncertainty Evaluation for Cali- bration Constant}

Calibration experiments are performed to derive the calibration constant $\alpha$. The total neutron yield is given as

$$
<S_{\mathrm{n}}>\text { [neutrons] }=\alpha \times<C>\text { [counts] }
$$

where $\left\langle S_{\mathrm{n}}>\right.$ the total neutron yield, $\langle C>$ the neutron detector count. To derive the total neutron yield from fusion 

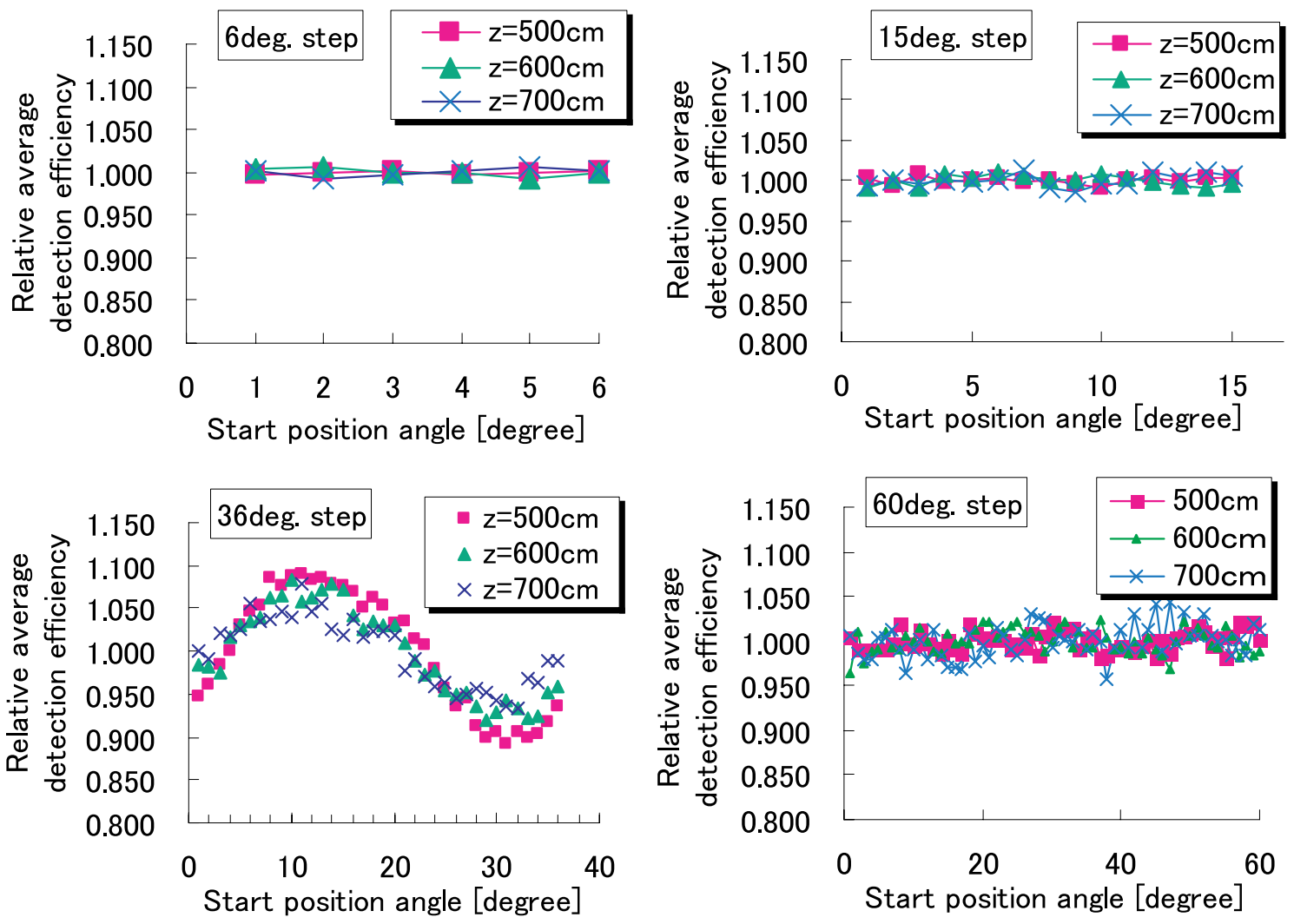

Fig. 3 Detection efficiency of the neutron detector placed on the center axis averaged over complete rotation. The relative average detection efficiencies to the average one calculated by fine step angle are calculated for various rotation step angles. The results calculated by varying the rotation start position angle are plotted.

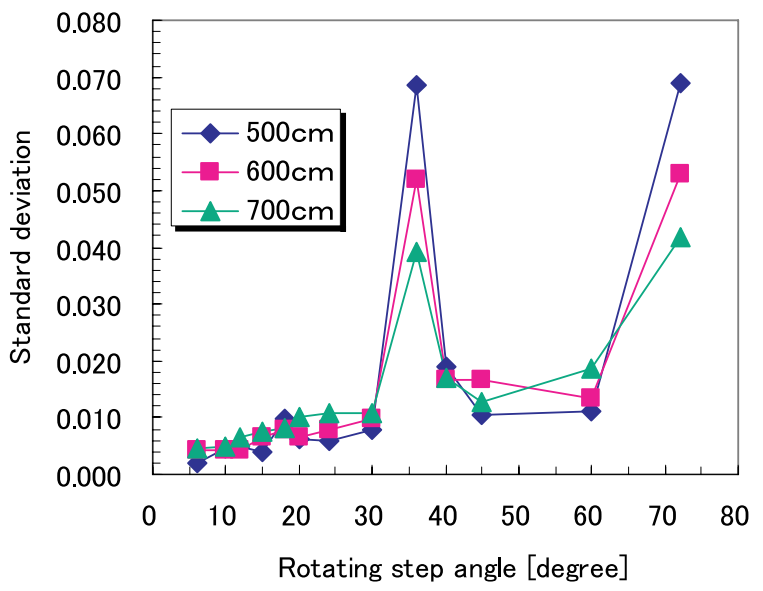

Fig. 4 Standard deviation of the relative average detection efficiency shown in Fig. 3 for various rotation step angles.

plasma, we should correct differences in neutron energy spectra and spatial distribution between the $\mathrm{Cf}$ source and the DD fusion plasma. The calibration constant for DD fusion neutrons is written as

$$
\alpha_{\mathrm{DD}}=\alpha_{\mathrm{Cf}} \times \frac{\varepsilon_{\mathrm{Cf}}}{\varepsilon_{\mathrm{DD}}},
$$

where $\alpha_{\mathrm{Cf}}$ calibration constant for Cf source neutrons, $\varepsilon_{\mathrm{DD}}$ the neutron detection efficiency for DD fusion plasma, $\varepsilon_{\mathrm{Cf}}$ the neutron detection efficiency for $\mathrm{Cf}$ point source. In the calibration experiments, only $\alpha_{\mathrm{Cf}}$ can be measured. $\varepsilon_{\mathrm{DD}}$ is calculated by MCNP code assuming that neutrons with $2.45 \mathrm{MeV}$ are emitted from plasma volume source. $\varepsilon_{\mathrm{Cf}}$ is also calculated by MCNP code assuming that neutrons with $\mathrm{Cf}$ fission spectrum are emitted from a point source rotating discretely.

The total uncertainty of the calibration constant is caused by statistical fluctuation caused when determining $\alpha_{\text {Cf }}$ in the calibration experiment, the certification uncertainty of the $\mathrm{Cf}$ standard source intensity and the uncertainty of the correction factor $\varepsilon_{\mathrm{DD}} / \varepsilon_{\mathrm{Cf}}$ caused when calculating the detection efficiency $\varepsilon_{\mathrm{DD}}$ and $\varepsilon_{\mathrm{Cf}}$ in MCNP code. We evaluate the total uncertainty of the $\alpha_{\mathrm{DD}}$ for the detectors placed at 500, 600 and $700 \mathrm{~cm}$ height on center axis. We assumed that total time for the calibration experiment is 9 hour/day $\times 7$ days and the neutron intensity of the $\mathrm{Cf}$ source is $10^{8} \mathrm{n} / \mathrm{s}$. Figure 5 shows the total uncertainty at various heights on the center axis. Uncertainties of each origin are listed in Table 1. The uncertainties of the detection efficiency for DD neutrons and for Cf source neutrons are less than $0.3 \%$ and $0.7 \%$, respectively. The statistical uncertainty in the calibration experiment is estimated to be less than $0.5 \%$. We assumed the uncertainty of the Cf source intensity was $1 \%$. The total uncertainties for all rotation step angles are less than $1.2 \%$ and increase with in- 
Table 1 Uncertainties of each origin contributing to the total uncertainty.

\begin{tabular}{ccccc}
\hline $\begin{array}{c}\text { Detector } \\
\text { height }(\mathrm{cm})\end{array}$ & $\varepsilon_{\mathrm{DD}}(\%)$ & $\varepsilon_{\mathrm{Cf}}(\%)$ & $\alpha_{\mathrm{Cf}}(\%)$ & $\begin{array}{c}\mathrm{Cf} \\
\text { intensity } \\
(\%)\end{array}$ \\
\hline \hline 500 & 0.24 & $0.30 \sim 0.47$ & 0.33 & \\
600 & 0.26 & $0.36 \sim 0.59$ & 0.41 & 1 \\
700 & 0.28 & $0.39 \sim 0.63$ & 0.44 & \\
\hline
\end{tabular}

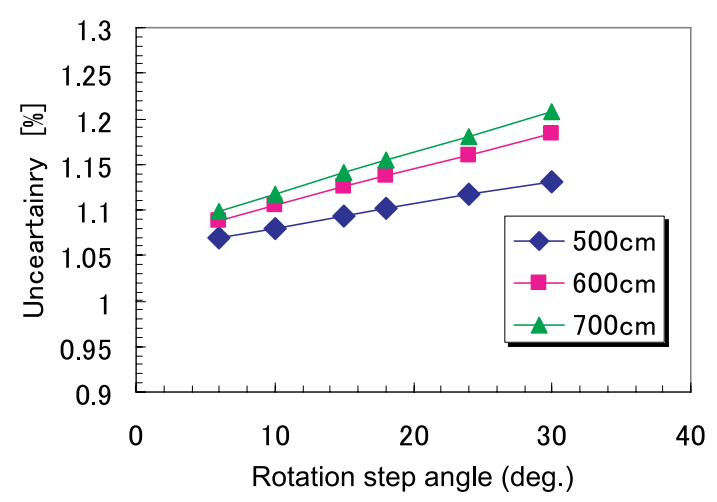

Fig. 5 Total uncertainty of the calibration constant $\alpha_{\text {DD }}$ for the detectors placed at 500, 600 and $700 \mathrm{~cm}$ height on the center axis.

creasing the step angle. This indicates that the rotation step angle of a $\mathrm{Cf}$ source can be relatively large for the neutron monitor placed on the center axis, because the sensitivity of the uncertainty on the rotation step angle is relatively small compared with the uncertainty of the source intensity.

\section{Summary}

We have calculated the detection efficiency of the neutron detector placed on center axis when ${ }^{235} \mathrm{U}$ fission chamber $\left({ }^{235} \mathrm{U}: 1.5 \mathrm{~g}\right)$ was adopted at LHD. The detection efficiency has 36 degree cyclic structures and the effect of the large support structure for the FIR laser infereometer.

We considered the source rotating procedure from the view point of the total calibration time and the statistical uncertainty of the calibration constant. The results show that the source locating precision can be rough because the cyclic structures can be canceled except for rotation step angles synchronizing with helical twist cycle.

We evaluate the total uncertainty of the calibration constant $\alpha_{\mathrm{DD}}$ for the detectors placed at 500, 600 and $700 \mathrm{~cm}$ height on center axis. The results indicate that the rotating step angle of a $\mathrm{Cf}$ source can be relatively large for the neutron monitor placed on the center axis.

\section{Acknowledgements}

This work is performed with the support and under the auspices of the NIFS Collaboration Research Program (NIFS08KOBH001 and NIFS10KLGA002).

[1] A. Iiyoshi, S. Imagawa and LHD Group, Fusion Eng. Des. 46, 323 (1999).

[2] O. Motojima, K. Akaishi et al., Nucl. Fusion 40, 599 (2000).

[3] J.F. Briesmeister (Ed.), MCNP - A General Monte Carlo Code for Neutron and Photon Transport Version 3A, (Los Alamos National Laboratory, New Mexico, 1986).

[4] N. Nishio, S. Yamamoto et al., Rev. Sci. Instrum. 81, 10D306 (2010). 